\title{
The Monte Carlo simulation of 2D ANNNI-model
}

Murtazaev Akai Kurbanovich ${ }^{1,2, a}$, Ibaev Zhavrail Gadzhievich. ${ }^{1, b^{*}}$

${ }^{1}$ Institute of Physics DSC RAS, Makhachkala, Yaragskogo 94, Russia

${ }^{2}$ Dagestan State University, Makhachkala, Gadzhieva 43a, Russia

aakai2005@mail.ru, beibaev77@mail.ru

Keywords: condensed state, competing interaction, modulated ordering, Fourier analysis.

\begin{abstract}
In this, study we present the data for 2D Axial Next Nearest Neighbor Ising model (ANNNI-model) obtained from Monte Carlo (MC) simulations using the standard Metropolis algorithm. The temperature dependences of thermodynamic parameters for a cubic lattice with linear sizes $L=32$ at different values of the competing interaction parameter $\left|J_{1} / J\right|=0.1 \div 1.0$. Transition temperatures of ferromagnetic ordering to the paramagnetic state at $\left|J_{1} / J\right|<0.3$ and to the modulated state at $0.3<\left|\mathrm{J}_{1} / \mathrm{J}\right|<0.5$ are shown to shift towards low temperatures with an increase in a competing interaction parameter absolute value. Conversely, transition temperatures of the modulate state to the paramagnetic ordering grow. The modulated ordering in the 2D ANNNImodel appears in the temperature range $0.1<\mathrm{T}<2.0$ at $0.2<\left|\mathrm{J}_{1} / \mathrm{J}\right| \leq 1.0$. Modulated structure parameters are computed using a mathematic apparatus of Fourier transform spectral analysis. According to the Fourier analysis results, the wave number grows with an increase in the competing interaction parameter absolute value. Summarizing obtained results, we plot a phase diagram of 2D anisotropic Ising model with competing interactions.
\end{abstract}

\section{Introduction}

Successes achieved in study of quasi-1D and quasi-2D transition metal-based magnetic materials are due in large part to the application of simplified multi-electron approaches by means of the Heisenberg and Ising models [1,2]. The Hamiltonians of these models have a fairly simple algebraic structure. This permits to prove a number of theorems concerning a character of precise energy spectrum and a spin character of the ground state, which have the considerable importance for testing the numerical simulation data [6-8]. However, as experience shows, main inferences

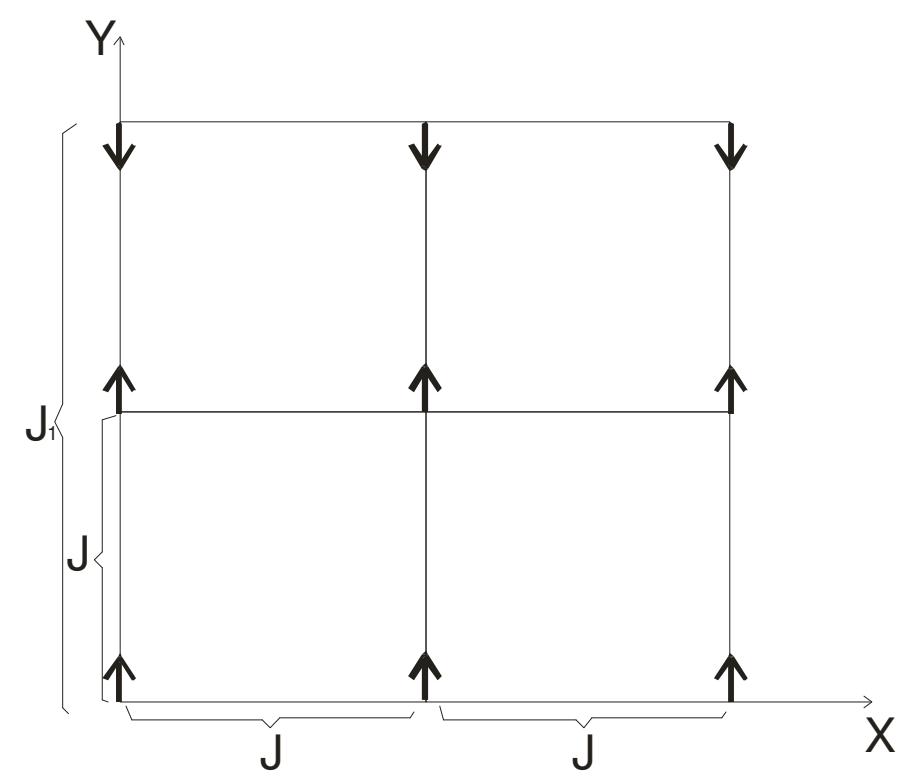

Fig. 1. The 2D ANNNI-model about a magnetic structure deduced within the framework of these simplified models are, as a rule, reserved when passing to stricter approaches too, for instance, which take into account the repulsion of electrons located in nonneighboring crystal lattice sites $[1,6]$.

The cubic Ising model with competing ferromagnetic and antiferromagnetic interactions (termed the ANNNI-model) was introduced to describe ordered magnetic phases in CeSb crystals [8]. A similar model is successfully used to evaluate the thermodynamics of oil microemulsions and is termed the Widom model [9]. In this study, we address 2D analog of such a model (Fig. 1), suitable for the description of microemulsion films on solid surfaces. It is assumed that

similar models can be used to optimize extraction processes, for instance, in an oil recovery.

The Hamiltonian of the model is 


$$
H_{A N N N I}=-J \sum_{i, j} s_{i} s_{j}-J_{1} \sum_{i} s_{i} s_{i+2},
$$

where $s_{i}= \pm 1, J>0$ is the parameter of nearest neighbor exchange interactions, $J_{1}<0$ is the parameter of next-nearest neighbor antiferromagnetic interaction along $\mathrm{Y}$ axis.

To define a phase behavior of the 2D ANNNI-model we employ approximate theoretical methods including high- and low-temperature splitting, the mean field theory, and other theoretical approximations.

According to literature data [8], when decreasing the temperature the ANNNI-model undergoes a second order transition from the paramagnetic to nearest ordered state, while the ferromagnetic to modulated phase transition, as we reported earlier, is of a first order [10].

\section{Methods and Results}

To address the thermodynamic and magnetic properties, we use the square systems of $L \times L$ sizes; $L=32$ with periodic boundary conditions. The number of spins in the simulated system $N_{\text {eff }}=1024$. The Monte Carlo simulations are performed by the Markov process of $\tau=1000 \tau_{0}$ in length $\left(\tau_{0}=10^{4} \mathrm{MC}\right.$ steps per spin, the length of non-equilibrium part). Thermodynamic parameters of the system are calculated by the averaging over one thousand different initial configurations along this chain. The estimation of an error for results estimated doesn't exceed sizes of symbols used in figures.
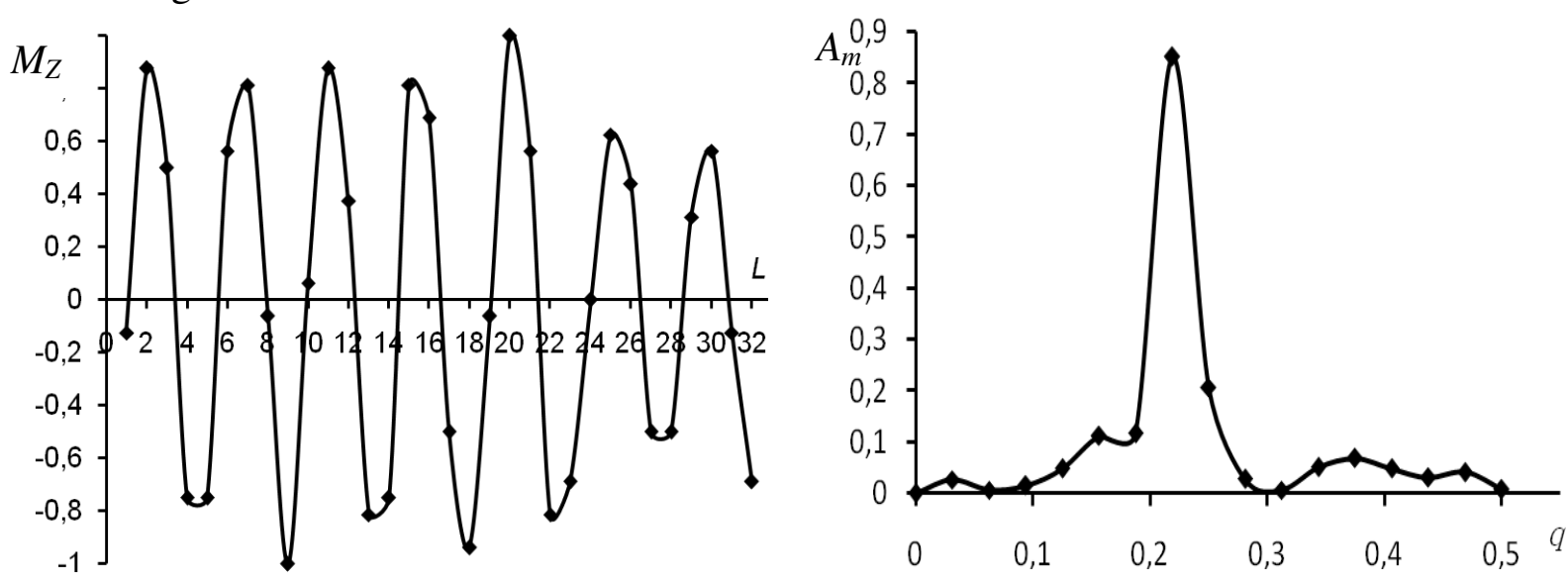

Fig. 2. The modulated structure of the $2 \mathrm{D}$ ANNNI-model at $L=32,\left|J_{l} / J\right|=1.0, t=1.95$ and the Fourier spectrum.

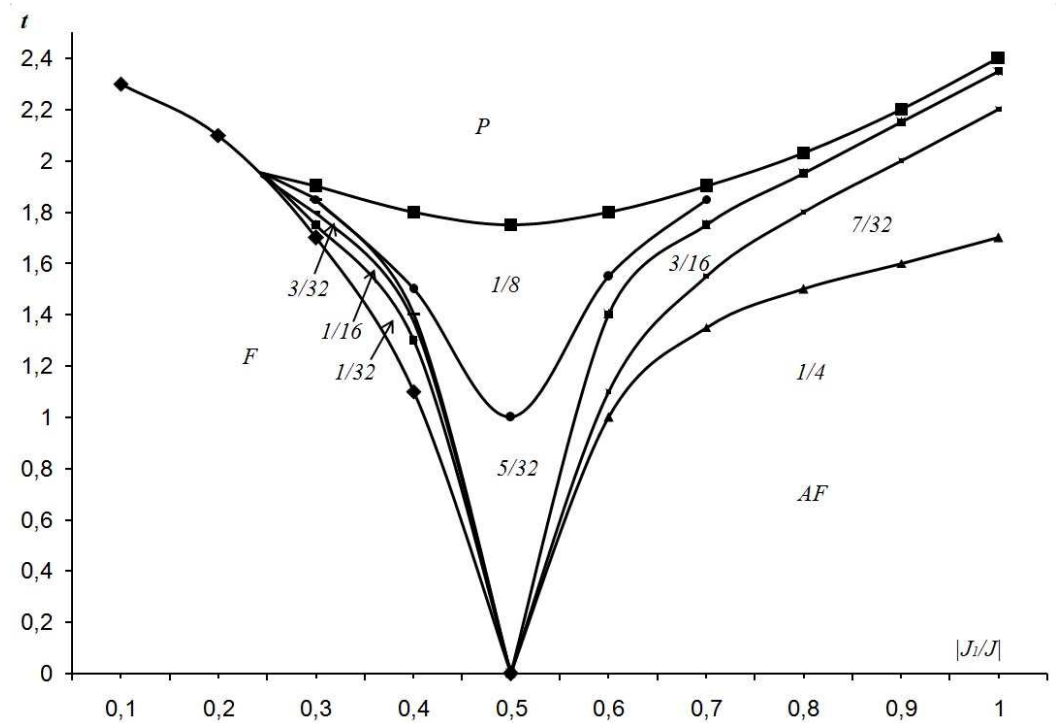

Fig. 3. The phase diagram of the $2 \mathrm{D}$ ANNNI-model ( $\mathrm{F}$ is ferromagnetic, $\mathrm{P}$ is paramagnetic, and $\mathrm{AF}$ is antiferromagnetic phases with $\mathrm{k}=1 / 4$, fractional numbers denote the values of the wave number $\mathrm{k}, \mathrm{t}=\mathrm{k}_{\mathrm{B}} \mathrm{T} / \mathrm{J}$ is the temperature). 
Magnetic properties of the modulated region are evaluated by means of a value

$$
M_{Z}=\frac{1}{L} \sum_{x}^{L} S_{x, y}
$$

which is the averaged magnetization of spin chains, perpendicular to $\mathrm{Y}$ axis. The averaged amplitude of the modulated structure is taken as the order parameter.

The parameters of modulated structures and transition temperatures between those are determined by a mathematic apparatus of Fourier spectral analysis

$$
M_{Z}=\frac{a_{0}}{2}+\sum_{k=1}^{N}\left[a_{k} \cos (2 \pi k z / L)+b_{k} \sin (2 \pi k z / L),\right.
$$

where

$$
\begin{aligned}
& a_{0}=\frac{1}{L} \sum_{z=1}^{L} M_{Z}, \\
& a_{k}=\frac{2}{L} \sum_{z=1}^{L} M_{Z} \cos (2 \pi k z / L), \\
& b_{k}=\frac{2}{L} \sum_{z=1}^{L} M_{Z} \sin (2 \pi k z / L), \\
& q=k / L,
\end{aligned}
$$

$k$ is the harmonic number, $\mathrm{z}$ is the layer number.

As the modulated structures are harmonic, the wave number $q$ is defined by the value of $k$, at which the amplitude function $A_{m}=\left(a_{k}^{2}+b_{k}^{2}\right)$ has a maximum.

Figure 2 depicts a typical ordering occurred in the $2 \mathrm{D}$ ANNNI-model at $\mathrm{L}=32,|\mathrm{~J} / \mathrm{J}|=1.0$, and temperature $t=1.95$ and a respective Fourier spectrum. As is easily seen from the figure, the model gains the modulated structure, which can be described by using the harmonic function

$$
M_{z}=A \cos (2 \pi q z+\varphi)
$$

where $A=0.749578$ is the amplitude of the modulated structure, $q=7 / 32$ is the wave number, $\varphi=3.14$ is the initial phase.

In Fig. 3, we plot a phase diagram of two-dimensional anisotropic Ising model with competing interactions (Fig. 3) employing the Fourier analysis results of modulated structures occurred at $\mathrm{L}=32$ and different temperatures and values of the competing interaction parameter. As is evident from the Fig.3, in the model, a region with the modulated ordering is observed at $0.2<\left|\mathrm{J}_{1} / \mathrm{J}\right| \leq 1.0$ in the temperature interval $0.1<\mathrm{T}<2.0$.

\section{Conclusion}

It is known that real magnetic systems comprise different physical and chemical interactions as well one or several kinds of the anisotropy. The full account of all possible anisotropy varieties and interactions results in the appearance of several types of the magnetic ordering and, as a consequence, of multicritical phenomena, which complicate a behavior and properties of the magnetic system. The complete description of solids requires a correct consideration of such processes and phenomena.

In this work, we investigate the model system with competing exchange interaction of nearest and next-nearest spins. The presence of the competing interaction is shown to lead to the occurrence of the modulated ordering in the system and the complication of thermodynamic and magnetic properties.

These results can be used for interpretation of real magnetic systems, where the modulated ordering is possible. However, we fail, at present, to find the literature data on real magnetic systems obtained experimentally in considered temperature ranges and values of exchange interaction constants. 


\section{References}

[1] A. A. Ovchinnikov, I. I. Ukrainskii, G. V. Kventsel', Theory of one-dimensional mott semiconductors and the electronic structure of long molecules having conjugated bonds, PhysicsUspekhi 15 (1973) 575-591

[2] S.R. White, Strongly correlated electron systems and the density matrix renormalization group, Phys. Reports 301 (1998) 187-204.

[3] I. Moreira, R. Dovesi, Periodic approach to the electronic structure and magnetic coupling in $\mathrm{KCuF}_{3}, \mathrm{~K}_{2} \mathrm{CuF}_{4}$, and $\mathrm{Sr}_{2} \mathrm{CuO}_{2} \mathrm{Cl}_{2}$ low-dimensional magnetic systems, Int. J. Quant. Chem. 99 (2004) 805-823.

[4] Hitesh J. Changlani, Norm M. Tubman \& Taylor L. Hughes, Charge density waves in disordered media circumventing the Imry-Ma argument, Scientific Reports 6 (2016) 3189731900.

[5] Zeph Landau, Umesh Vazirani \& Thomas Vidick, A polynomial time algorithm for the ground state of one-dimensional gapped local Hamiltonians, Nature Physics 11 (2015) 566-569.

[6] M. A. Shakirov, S. V. Tsibulsky, A. E. Antipov, Y. E. Shchadilova \& A. N. Rubtsov, Modeling the metastable dynamics of correlated structures, Scientific Reports 5 (2015) 8005-8008.

[7] D.J. Klein, Ground-state features for Heisenberg models, Chem. Phys. 77 (1982) 3098-3100.

[8] R.J. Elliott, Phenomenological discussion of magnetic ordering in the heavy rare-earth metals, Phys. Rev. 124 (1961) 346-353.

[9] B. Widom, Lattice model of microemulsion, J. Chem. Phys. 84 (1986) 6943-6954.

[10] A.K. Murtazaev, J.G. Ibaev, Critical properties of an ANNNI-model in the neighborhood of multicritical Lifshitz point, Solid State Communications 152 (2012) 177-179. 